\title{
A rare case of Rosai-Dorfman disease without lymphadenopathy
}

\author{
Hyerim $\mathrm{Ha}^{1}$, Ki Hwan $\mathrm{Kim}^{2}$, Young Joon $\mathrm{Ahn}^{3}$, Ji Hye Kim${ }^{4}$, Ji Eun Kim², and Sung-Soo Yoon ${ }^{1}$
}

\begin{abstract}
${ }^{1}$ Division of Hematology and Medical Oncology, Department of Internal Medicine, Seoul National University Hospital, Seoul; ${ }^{2}$ Division of Hematology and Medical Oncology, Department of Internal Medicine, ${ }^{3}$ Department of General Surgery, Seoul Metropolitan Government Seoul National University Boramae Medical Center, Seoul; ${ }^{4}$ Department of Internal Medicine, Seoul National University Hospital, Seoul; ${ }^{5}$ Department of Pathology, Seoul Metropolitan Government Seoul National University Boramae Medical Center, Seoul, Korea
\end{abstract}

\section{Received: February 2, 2015 \\ Revised : March 9, 2015 \\ Accepted: April 15, 2015}

\section{Correspondence to \\ Ki Hwan Kim, M.D.}

Division of Hematology and Medical Oncology, Department of Internal Medicine, Seoul

Metropolitan Government Seoul

National University Boramae

Medical Center, 20 Boramae-ro

5-gil, Dongjak-gu, Seoul 07o61,

Korea

Tel: +82-2-870-2229

Fax: +82-2-870-1476

E-mail: floresta405@gmail.com

\section{To the Editor,}

Rosai-Dorfman disease (RDD) was first described by Rosai and Dorfman [1] in 1969 including four cases. In 1990, 433 cases with massive lymphadenopathy were described. RDD is a rare histiocytic proliferative disorder that occurs in children and young adults that mean age at the time of diagnosis is 20.6 years. Patients commonly present with massive, painless lymphadenopathy, fever, malaise, night sweats, neutrophilia, high erythrocyte sedimentation rate (ESR), and polyclonal hypergammaglobulinemia [2].

Extranodal manifestation is common and seen in up to $43 \%$ of patients. The skin is the most common extranodal site of involvement (27\%) followed by the soft tissue of the nasal cavity. Only $15 \%$ of patients have splenic abnormalities. Furthermore, most of them have only mild splenomegaly on physical examination. Only a single case of splenic involvement with hypereosinophilic syndrome has been reported [3]. There are no literature regarding the histologically proven isolated spleen and liver involvement without lymphadenopathy in RDD.

The etiology and pathogenesis of RDD are unclear. The hypotheses include immune regulation disorder or infection, such as various herpes viruses, Epstein-Barr virus (EBV), cytomegalovirus (CMV), Brucella, or Klebsiella.
Several infective agent or antigen can cause proliferation of histiocytes, and the key factor is thought to be macrophage colony-stimulating factor. Lymph node histology shows clonal histiocytes in all forms and the massive sinus infiltration of histiocyte-macrophage cells. It has a positive staining for S-100, alpha-1 antichymotrypsin, and CD68 antigens while negative staining for CDia [4].

Many cases are self-limiting and have indolent clinical courses. There is no ideal treatment for the disease. Onehalf of the patients have spontaneous resolution. Surgery is a treatment modality for symptomatic disease such as airway compression or nasal obstruction. Multiorgan involvement or association with immune dysfunction is an indication of systemic treatment.

Several modalities of treatment have been used, such as radiotherapy, chemotherapy, and glucocorticoid therapy. There is no standard chemotherapy regimen; however, the most common chemotherapeutic agents are methotrexate, 6-mercaptopurine, and cladribine or vinorelbine plus methotrexate. Interferon treatment has been reported also [5]. The progress of the disease is variable. Regular follow-up is important in the management of these patients.

A 58-year-old woman admitted to be evaluated for anemia in May 2013. The patient presented with dyspnea 


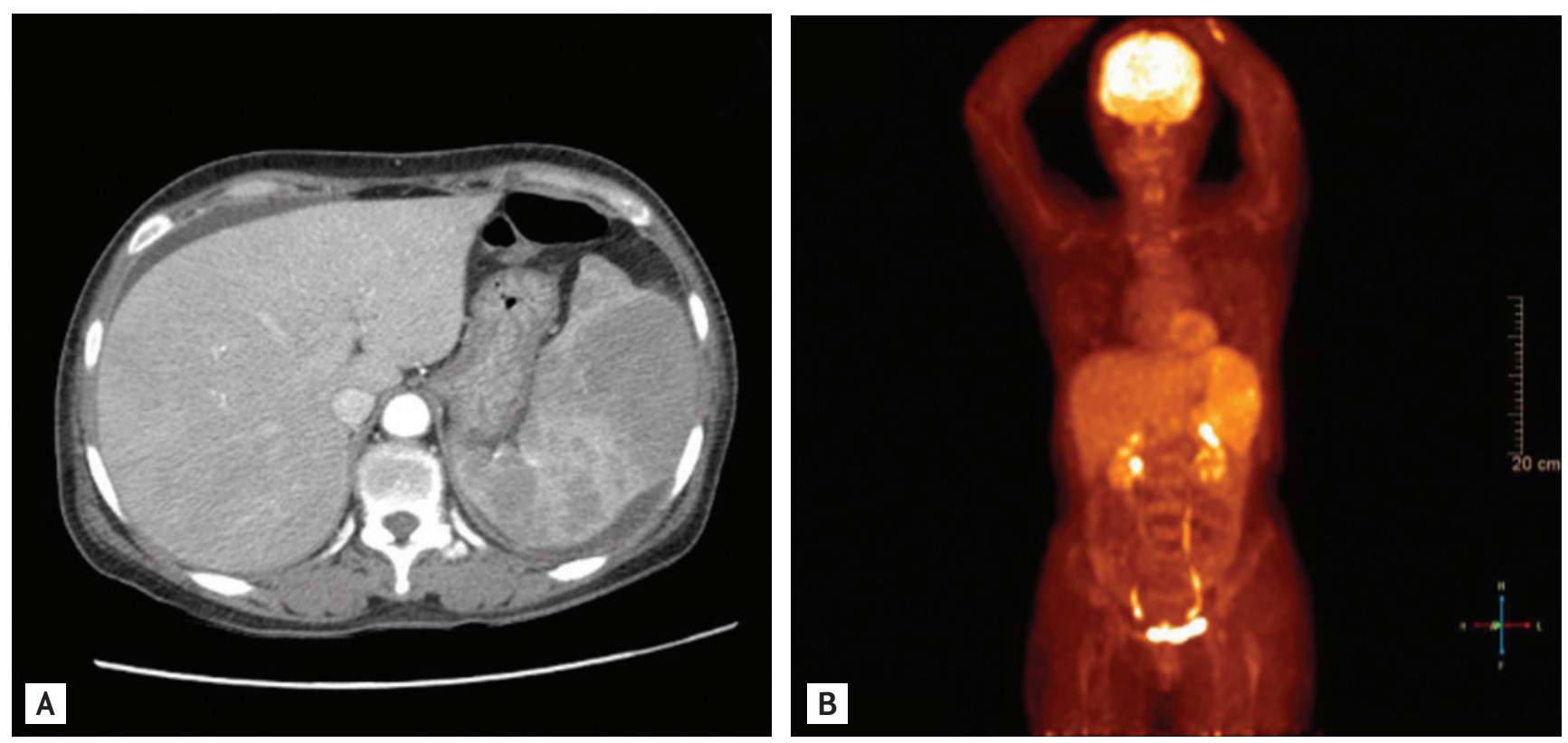

Figure 1. Abdominal computed tomography \& fluorodeoxyglucose-18 positron emission tomography. (A) Hepatosplenomegaly with heterogeneous enhancement of the liver and spleen. (B) No hypermetabolic lesion except for an enlarged spleen.

and cramping pain in both hands, which lasted for one month. She did not have fever, weight loss, night sweats, myalgia, or arthritis. She is never smoked, social drinker, and was not on any medication except for an iron supplement. A physical examination revealed multiple purple bruises and hepatosplenomegaly without peripheral lymph node enlargement.

A blood laboratory test revealed anemia of $4.7 \mathrm{~g} / \mathrm{dL}$ that had decreased rapidly from $7.8 \mathrm{~g} / \mathrm{dL} 1$ month prior. The reticulocyte fraction was $10.2 \%$. The platelet count was severely decreased to $22,000 / \mu \mathrm{L}$, and it was refractory to repeated transfusions. A peripheral blood smear showed macrocytic hypochromic red blood cells, anisocytosis, polychromasia, poikilocytosis, and teardrop cells. Iron, vitamin $\mathrm{B} 12$, and folate measured $4 \mathrm{O} \mathrm{g} / \mathrm{dL}$ (normal range, 50 to 130 ), $159 \mathrm{pg} / \mathrm{mL}$ (normal range, 200 to 950 ), and 8.12 $\mathrm{ng} / \mathrm{mL}$ (normal range, 3 to 17 ), respectively. Total bilirubin was slightly elevated at $1.9 \mathrm{mg} / \mathrm{dL}$ (normal range, 0.2 to 1.2). ESR was $5 \mathrm{~mm} / \mathrm{hr}$ (normal range, o to 20). Human immunodeficiency virus antigen/antibody and serologic test for hepatitis were negative. EBV antigens were also negative. CMV, herpes virus, bacterial test, and immunoglobulin in serum were not performed initially.

Abdominal computed tomography showed hepatosplenomegaly with heterogeneous enhancement of the liver and multiple nodules of the spleen. Abnormal lymphadenopathy was absent (Fig. 1A). On bone marrow aspiration and trephine biopsy, hypercellular marrow with reactive erythroid hyperplasia was observed. Fluorodeoxyglucose-18 positron emission tomography revealed no hypermetabolic lesion except for an enlarged spleen with diffuse hypermetabolism (Fig. 1B). Magnetic resonance imaging of the brain was normal.

The patient underwent splenectomy. The cut surface revealed multifocal yellowish-brown or mahogany-colored patchy lesions without well-defined masses. On low-power histologic examination, the spleen showed a multifocal hemorrhagic infarct with diffuse infiltration of pale-staining plump cells. These large histiocytic cells were characterized by emperipolesis, the engulfment of intact lymphocytes or red blood cells (Fig. 2A and 2B).

Immunohistochemically, these cells were negative for CD1a (Fig. 2C) and positive for CD68 (Fig. 2D) and S-100 (Fig. 2E), which was consistent with RDD. EBV was not detected in the specimen by Epstein-Barr encoding region in situ hybridization. Hematologic deterioration due to hypersplenism, such as hemolytic anemia with reticulocytosis and thrombocytopenia, dramatically improved after splenectomy.

In the current case, we presented a rare case of RDD that showed only spleen and liver involvement without lymphadenopathy. Splenectomy alone could be both a 

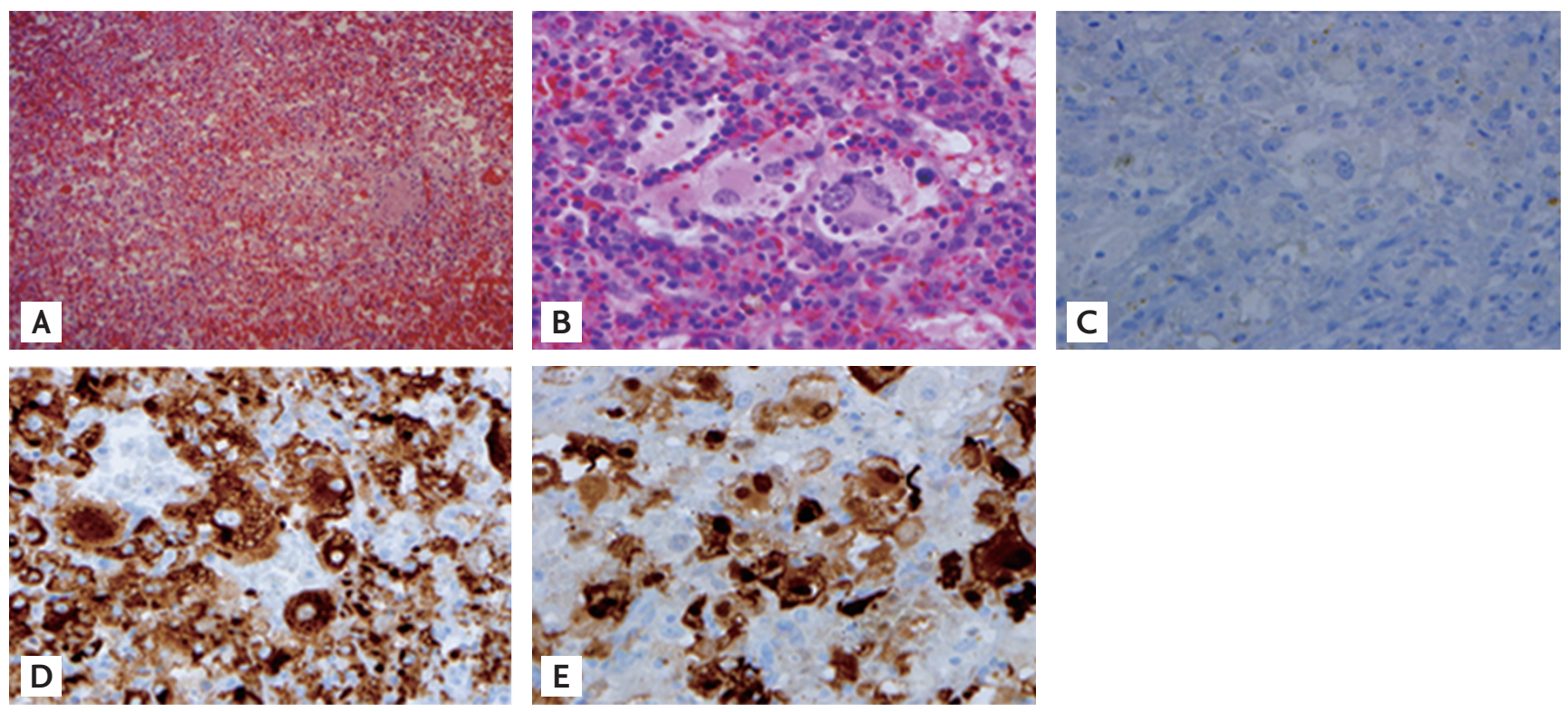

Figure 2. Pathology. (A, B) Hemorrhagic infarction, large histiocytes with the engulfment of intact lymphocytes or red blood cells $(A, \times 100 ; B, \times 400)$. (C) Immunohistochemical stain, CD1a negative ( $\times 400)$. (D) Immunohistochemical stain, CD68 positive $(\times 400)$. (E) Immunohistochemical stain, S-100 positive $(\times 400)$.

diagnostic and therapeutic approach in such a patient.

Keywords: Histiocytosis, sinus; Hepatomegaly, splenomegaly; Lymphatic disease

\section{Conflict of interest}

No potential conflict of interest relevant to this article was reported.

\section{REFERENCES}

1. Rosai J, Dorfman RF. Sinus histiocytosis with massive lymphadenopathy: a newly recognized benign clinicopathological entity. Arch Pathol 1969;87:63-70.

2. Bernacer-Borja M, Blanco-Rodriguez M, Sanchez-Grana-
dosJM, Benitez-Fuentes R, Cazorla-Jimenez A, Rivas-Manga C. Sinus histiocytosis with massive lymphadenopathy (Rosai-Dorfman disease): clinico-pathological study of three cases. Eur J Pediatr 2006;165:536-539.

3. Hernandez D, Gutierrez L, Duque H, Olavarria R, Muci R, Wunaii H. Association of sinus histiocytosis with massive lymphadenopathy and idiopathic hypereosinophilic syndrome. Histol Histopathol 1987;2:239-242.

4. Carbone A, Passannante A, Gloghini A, Devaney KO, Rinaldo A, Ferlito A. Review of sinus histiocytosis with massive lymphadenopathy (Rosai-Dorfman disease) of head and neck. Ann Otol Rhinol Laryngol 1999;108:10951104 .

5. Lohr HF, Godderz W, Wolfe T, et al. Long-term survival in a patient with Rosai-Dorfman disease treated with interferon-alpha. Eur J Cancer 1995;31A:2427-2428. 